\title{
Switching-on and -off dynamics of MQW structures with bistable electro-optical absorption
}

\author{
V. A. Kochelap \\ Institute of Semiconductor Physics, NAS Ukraine, 45 prospekt Nauki, Kyiv, 252028, Ukraine
}

\author{
L. L. Bonilla and C. A. Velasco \\ Universidad Carlos III de Madrid, Escuela Politcnica Superior, Butarque 15, E-28911 Legans, Spain
}

\begin{abstract}
In this work we have studied the dynamics of switch-on and switch-off processes in biased MQW structures where every well shows optical bistability in a light intensity range $\left(I_{l}, I_{h}\right)$. We have analyzed in detail MQW structures with negligible inter-well transport. We have found that the switch-on mechanism consists of a time sequence where every QW jumps into the highabsorption state. Therefore a step-like switching wave propagates through the structure. The switchoff process resembles a reverse wave propagating in the opposite direction and step-like processes in the plasma concentration decay. These effects can be used for conversion of an analog optical signal to digital (optical and electrical) signal(s).
\end{abstract}

Keywords: optical bistability, multiple quantum well heterostructures

Paper received 08.05.98; revised manuscript received 25.06.98; accepted for publication 27.10.98.

There are many quantum well heterostructures that show bistable electro-optical absorption: multiple quantum well heterostructures placed into the intrinsic region of a p-i-n diode connected to an electric circuit with a series resistor (Self Electro-optic Effect Devices, SEEDs) [14]; similar structures with an open circuit $[5,6]$; multiple quantum well structures placed between charged capacitor plates [7-9]; staked asymmetric double and triple QWs [10], and others [11]. The bistable absorption arises due to the screening of the applied field by the photo-generated electrons and holes, which produces considerable changes in the optical spectra near the fundamental edge of absorption. These spectra become dependent on the concentration of the electron-hole plasma, i.e., on the intensity of the illumination. If the spectrum of the illuminating light is tuned into the region between exciton and interband absorption, the light absorption becomes bistable; i.e. for a given range of incident light intensities both, low absorption (LA) state with low plasma concentration, and high absorption (LA) state with large plasma concentration can exist.

In these systems the dynamics of the switching processes between bistable states involves different physical processes: generation of excitons associated with the two-dimensional electron and hole subbands, fast exchange between exciton and electron-hole states, intra- well separation of electrons and holes, changes in their wave functions, and inter-well transport. Understanding these processes and their manifestation in optics is important for both, physics and its applications. In this work we study the dynamics of switch-on and -off processes in a voltage biased MQW structure without inter-well electron transfer, i.e., with an independent balance of the photo-excited plasma concentration for every QW. Electro-optical bistability in such structures was investigated in papers [7-9] under quasi-stationary conditions.

A model describing switching processes in optically bistable MQW structures should include a self-consistent calculation of electron-hole states in the wells (particularly subband energies, $E_{e}, E_{h}$, and wave functions, $\Psi_{e}$, $\Psi_{h}$ ), the absorption factor $A$, and exciton and plasma concentrations $(N)$. Two groups of characteristic time scales are important for the dynamics of switching. The first group comprises faster processes with characteristic quantum mechanical times $\hbar / E_{e}, \hbar / E_{p}$, and exchange times between exciton and electron-hole states, $\tau_{e x}$. The second group of time scales is related to the generation and recombination of the photo-generated carriers. These time scales can be estimated as $N_{c h} / A I$ and $\tau_{R}$, where $N_{c h}$ is a characteristic plasma concentration, $I$ is a typical light intensity, and $\tau_{R}$ is the recombination time. The time 


\section{A. Kochelap et al.: Switching-on and -off dynamics of MQW structures ...}

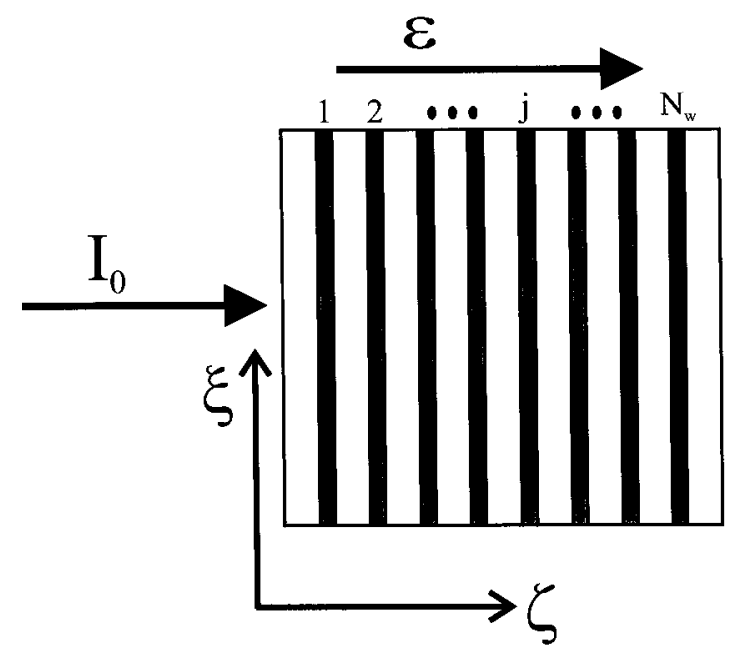

Fig. 1. Scheme of a MQW heterostructure illuminated by an incident light of intensity $I_{0}$ and biased by an electric field $E$.

scales in the first group are considerably smaller than those in the second group. Then the dynamics of the system can be described by means of electron-hole states calculated at instantaneous plasma concentration $N(t)$. This results in an exciton energy $E_{e r}$, which follows the position of the electron and hole subbands, and an absorption factor adiabatically dependent on $N(t)$. This approach is valid for light pulses with a duration of a few picoseconds or longer.

Let us consider a MQW structure biased by an electric field and illuminated from below, as illustrated in fig. 1. The QWs have width $2 d$, and are grown vertically along the $\varsigma$ axis ( $\varsigma$ is expressed in units of $d$ ). Introducing the characteristic energy $E_{0}=\hbar^{2} / 2 m d^{2}$, we will measure subband energies and potential energies in these units, while the electric field is in units of $\varepsilon_{0}=E_{0} / e d$. The dimensionless electron and hole concentrations $(n)$ are in units of $N_{0}=\kappa E_{0} / e^{2} d, \kappa$ being the dielectric permitivity. The $k^{\text {th }} \mathrm{QW}\left(k=1 \ldots N_{\omega}, N_{\omega}\right.$ is the total number of QWs) can be described by its dimensionless electron and hole wave functions $\psi_{e_{k}}, \psi_{h_{k}}$, subband electron and hole energies $\varepsilon_{e_{k}}$ and $\varepsilon_{h_{k}}$ (only the lowest subbands are supposed to be populated), and concentration of the photo-excited electron-hole plasma (supposedly quasineutral) $n_{k}$. We also assume that electrons and holes have equal effective masses for simplicity. According to the above discussion, the wave functions and energies of the $k^{\text {th }}$ QW can be found from the self-consistent Schrodinger-Poisson equation at a given plasma concentration $n_{k}$

$\frac{d^{2} \psi_{k}}{d \zeta^{2}}+\left(\varepsilon_{k}+v_{k}-q \zeta\right) \psi=0$

$\psi_{k}( \pm 1)=0, \quad \int_{-1}^{1}\left|\psi_{k}\left(\zeta ; n_{k}\right)\right|^{2} \zeta=1$
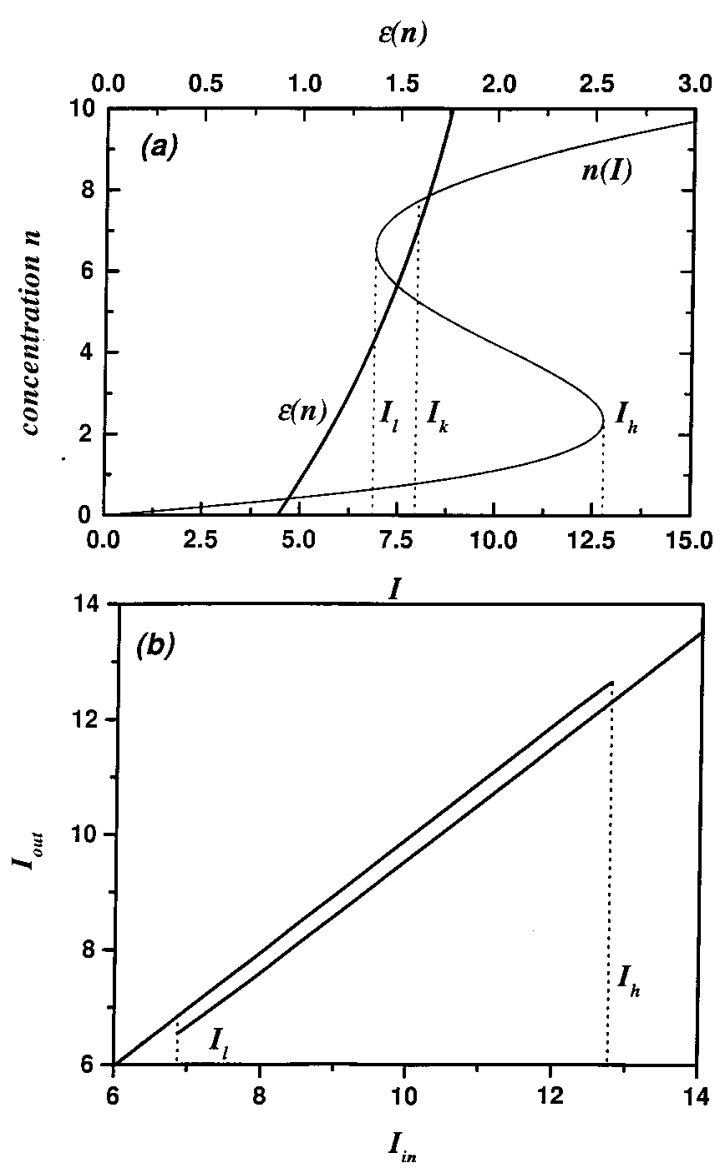

Fig. 2. Steady state characteristics of individual QWs. (a) shows the renormalized energy $\varepsilon$ (scale on top of the figure) as function of the plasma concentration $n$ (left axis), and bistable characteristic curve $n(I)$ : plasma concentration as function of the incident light intensity $I$ (left and bottom axis). (b) shows the hysteresis in the transmitted light through a single QW.

where $q$ is the dimensionless applied electric field, and the dimensionless electrostatic energy is

$v_{k}\left(\zeta ; n_{k}\right)=n_{k} \int_{-1}^{1} \zeta^{\prime} K\left(\zeta, \zeta^{\prime}\right)\left|\psi_{k}\left(\zeta^{\prime} ; n_{k}\right)\right|^{2}$

$K\left(\zeta, \zeta^{\prime}\right) \equiv \frac{1}{2}\left(\left|\zeta-\zeta^{\prime}\right|-\left|\zeta+\zeta^{\prime}\right|\right)$

The electrostatic energy and the wave functions have the following symmetry properties: $v_{k}\left(\zeta ; n_{k}\right)=-v_{k}\left(-\zeta ; n_{k}\right)$, and $\psi_{e_{k}}\left(-\zeta ; n_{k}\right)=\psi_{h_{k}}\left(-\zeta ; n_{k}\right)=\psi_{k}\left(\zeta ; n_{k}\right)$. The eigenvalue $\varepsilon_{k}$ depends parametrically on $n_{k}$. We have found solutions of equation (1) by means of a variational method. The energy $\varepsilon_{k}$ as a function of $n_{k}$ for a particular value of the electric field $q$ is presented in fig. 2(a). Parameters used in the calculations are given in table. The increase in the electron and hole energies with carrier concentration arises, obviously, from the screening of the applied field.

In case of deep QWs and large exciton radius, the 


\section{A. Kochelap et al.: Switching-on and -off dynamics of MQW structures ...}

exciton energy $E_{e x}$ follows the position of the electron and hole subbands. Assuming a Lorentz shape for the absorption factor as function of the photon energy $\hbar \omega$, we can write:

$A\left(n_{k}, q, \omega\right)=\frac{A_{m} \Lambda^{2}}{\left(\varepsilon_{k}\left(n_{k}, q\right)-\Delta\right)^{2}+\Lambda^{2}} \equiv$
$\equiv A_{m} a\left(n_{k}, q, \omega\right)$

where $A_{m}$ is the maximum absorption factor, $\Delta=\left(\hbar \omega+E_{e x}-E_{g}\right) / 2 E_{0}$ is the detunning of the photon energy, and $\Lambda$ is the dimensionless bandwidth in units of $2 E_{0}$.

We can describe the MQW structure in the case of independent concentration balance for each QW by the following equations:

$\frac{d n_{k}}{d t}=a\left(n_{k}, q, \omega\right) I_{k-1}-n_{k} \equiv$
$\equiv R\left(n_{k}, q, \omega, I_{k-1}\right)$,

$I_{k}=\left(1-A_{m} a\left(n_{k}, q, \omega\right)\right) I_{k-1}, \quad k=1 \ldots N_{\omega}$.

Here we assume a linear recombination rate. $t$ is measured in units of the recombination time $\tau_{R}$. For some particular parameters $\omega$ and $q$, the stationary dependence $n(I)$ is presented in fig. 2. $I_{k-1}$ is the illuminating $k^{\text {th }}$-QW layer intensity in units $I_{0} \stackrel{k-1}{=} N_{0} / \tau_{R} A_{m}$. Thus, $I_{0}(t)$ is the incident light intensity. The instant relationship (4) between $I_{k}(t)$ and $I_{k-1}(t)$ requires the condition $\frac{1}{I_{k}(t)} \frac{d I_{k}}{d t}<<\frac{c(1-a)-\tau_{R}}{2 d}, c$ being the velocity of light. This relation holds for pulses with a duration of a few picoseconds or longer. For a given $I_{0}(t)$, and an initial concentration in every well $n_{k}(0)$, the system of equations (1) and (2) defines completely the dynamic problem for the MQW structure.

Table. Numerical values used for calculation.

\begin{tabular}{lc}
\hline Parameter & Value \\
$E_{0}(\mathrm{meV})$ & 5.6 \\
$\varepsilon_{0}(\mathrm{kV} / \mathrm{cm})$ & 5.6 \\
$N_{0}\left(\mathrm{~cm}^{-2}\right)$ & $4.0 \times 10^{10}$ \\
$I_{0} / \hbar \omega\left(\right.$ photons $\left./ \mathrm{cm}^{2} \mathrm{~s}\right)$ & $8.1 \times 10^{21}$ \\
\hline \hline
\end{tabular}

Let us consider the steady state condition. For this case, the equation

$R\left(n_{k}, q, w, I_{k-1}\right)=0$

gives the possible solutions for the $k$ th $\mathrm{QW}$. It is easy to see that for the absorption factor of (6) there are three branches of uniform solutions $n_{k}=n_{k}(I, q)$ at some intervals of intensities $I$ and fields $q$ : the low absorption branch [low concentration $n_{L}(I)$ ], the high absorption branch [high concentration $\left.n_{H}(I)\right]$ and the middle branch (which is unstable). In fig. 2(b) we present these branches calculated for an individual QW for the particular parameter values of table. The bistable regime occurs in the interval $I_{l}<I<I_{h}$. The light intensity transmitted through the $k$ th QW layer shows hysteresis, as depicted in fig. 2(b).

Using these solutions for an individual QW, we can easily construct possible steady states for the whole MQW structure. If the incident intensity $I_{0}$ is less than the value $I_{l}$, the MQW structure is entirely in the LA state. If $I_{l}<I_{0}<I_{h}$, the whole structure can be in the LA state, or some of the QWs adjacent to the illuminated face can switch to the HA state. At $I_{0}>I_{h}$ the very first QW layers are certainly in the HA state, while the rest of wells can be in the LA state. In fig. 3 the intensities $I_{k}$ as function of the index $k$ are presented for a 24-QW structure at $I_{0}=1.25 \times I_{h}$. The first seven QWs are always in the HA state and the intensity decreases fast across this region of the structure. The QWs with $k$ from 8 to 20 can be in both states. The QWs with $k>20$ are always in the LA state. This results in thirteen possible stationary states of the MQW structure. Three of these possible states are shown

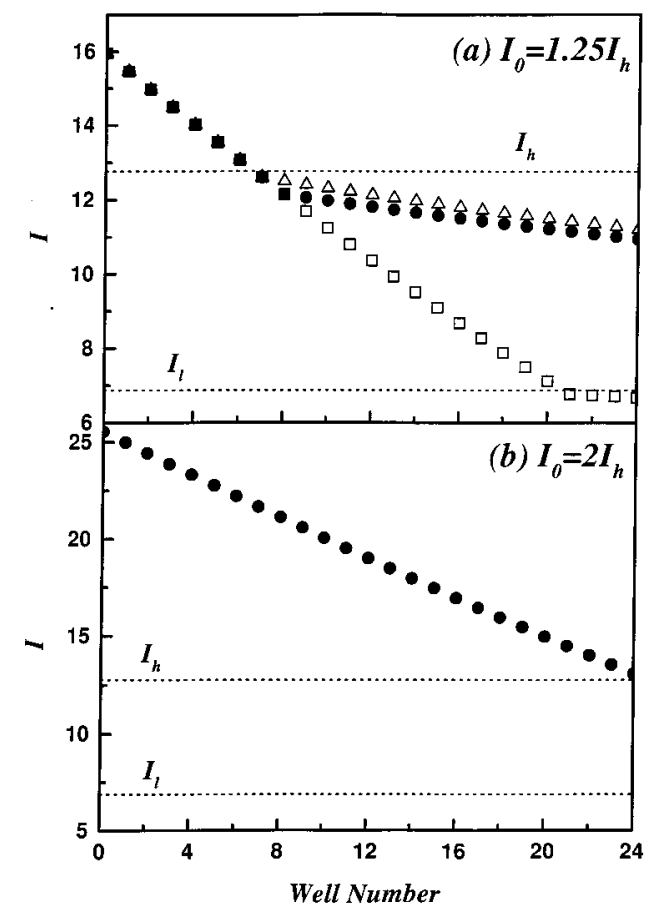

Fig. 3. Distribution of intensities across the MQW structure. Positions of individual QWs are indicated. (a) corresponds to an incident light $I_{0}=1.25 I_{h}$. Three of the possible thirteen steady states described in the text are shown. Triangles correspond to the case (i), circles correspond to the case (ii) and squares correspond to the case (iii). (b) shows the stationary situation when $I_{0}=2 I_{h}$. All the wells are in the HA state. 


\section{A. Kochelap et al.: Switching-on and -off dynamics of MQW structures ...}

in the fig. 3(a): (i) seven QWs in the HA state and the rest in the LA state, (ii) eight QWs in the HA state and the rest in the LA state, and (iii) the wells with $k=1 \ldots 20$ are in the HA state, while the rest are in the LA state. The larger is the intensity of the incident light, $I_{0}$, the larger will be the number of QWs switched to the HA state. There is a critical value of the incident light intensity $I_{0}^{*}$, dependent on the number of QWs of the structure, such that if $I_{0} \geq I_{0}^{*}$, all the wells are switched to the HA state. Fig. 3(b) depicts such a case for a 24QW structure when $I_{0}=2 I_{h}$. Thus, the bistability of the optical absorption in a single QW, generally leads to multistability in the MQW structure. Which particular state occurs depends on the prehistory, i.e., on the timedependence of the incident intensity, $I_{0}(t)$, during the build-up of the stationary value ${ }^{1}$.

Let us go back to the dynamical problem and consider a time-dependent intensity of the incident light. We introduce the characteristic time scale of the build-up of the stationary light intensity value $\tau_{i n}$, so that

$I=I\left(\frac{\tau_{R}}{\tau_{\text {in }}} t\right)$. If

$\beta \equiv \frac{\tau_{R}}{\tau_{\text {in }}}<<1$,

we can perform a perturbative analysis. Let us rescale (3) introducing the new time variable: $\bar{t} \equiv \beta t$. Then we obtain that this equation can be rewritten as:

$\beta \frac{d n_{k}}{d \bar{t}}=a\left(n_{k}, q, \omega\right) I_{k-1}-n_{k} \equiv$

$\equiv R\left(n_{k}, q, \omega, I_{k-1}\right)$

Under condition (2), we can apply the boundary layer singular perturbation method to solve (8) (see, for example [12]). According to this method, the solution evolves smoothly over time intervals which last periods $\bar{t} \equiv O(1)$ separated by sharp transitions lasting times $\bar{t} \equiv O(\beta)$. Outer solutions evolving on the long time scale $\bar{t}$ can be found by treating the term multiplied by the small parameter $\beta$ as a perturbation:

$n_{k}(\bar{t})=n_{k, 0}(\bar{t})+\beta n_{k, l}(\bar{t})+O\left(\beta^{2}\right)$.

${ }^{1}$ If carriers may hop between QWs, the steady state distribution is, in principle, determined by this transfer. Let $t_{i n t}$ be the characteristic time of the inter-well transport. A suppressed interwell carrier transfer means $\tau_{R} / \tau_{\text {int }}<<1$. Thus, the results of our analysis are valid for $\frac{1}{I} \frac{d I}{d \bar{t}}>>\frac{\tau_{R}}{\tau_{\text {int }}}$. If the latter inequality is not satisfied, the results are valid for times $t<<\tau_{\text {int }} / \tau_{R}$.
Here the leading term $n_{k, 0}(\bar{t})$ coincides with one of the two branches of the solutions of $(6), n_{L}(I), n_{H}(I)$, with $\bar{t}$-dependent $I=I_{k-1}$. The first correction to the outer solution is determined by

$$
\begin{aligned}
& n_{k, 1}(\bar{t})=-\frac{1}{\left(\frac{\partial a\left(n_{k, 0}\right)}{\partial n_{k, 0}} I_{k-1}(\bar{t})-1\right)^{2}} \times \\
& \times \frac{d I_{k-1}(\bar{t})}{d \bar{t}} a\left(n_{k, 0}\right)
\end{aligned}
$$

If the leading term lies on the low concentration branch, $n_{k, 0}=n_{L}\left[I_{k-1}(\bar{t})\right], I_{k-1} \leq I_{h}$, the first correction is small everywhere except in a vicinity of $\bar{t}=\bar{t}_{k, h}$, where $I_{k-1}\left(\bar{t}_{k, h}\right)=I_{h}$ at $(I / \bar{t})_{\bar{t}_{k-1, h}}>0$. As $\bar{t} \rightarrow \bar{t}_{k, h}$ the denominator term in (10) goes to zero. Similarly, when the leading term lies on the high concentration branch, $n_{k, 0}=n_{H}\left[I_{k-1}(\bar{t})\right], I_{k-1} \leq I_{l}$ the first correction is small everywhere, except in a vicinity of $\bar{t}=\bar{t}_{k, l}$, with $I_{k-1}\left(\bar{t}_{k, l}\right)=I_{l},(I / \bar{t})_{\bar{t}_{k-1, l}}<0$. For increasing $I_{k-1}(\bar{t})$ near $\bar{t}=\bar{t}_{k, h}$, and for decreasing $I_{k-1}(\bar{t})$ near $\bar{t}=\bar{t}_{k, l}$, the jumps between low concentration and high concentration solutions are described by inner solutions on the fast time scale $t$. To find the latter solutions we can keep $I_{k-1}(\bar{t})=I_{h}$ or $I_{k, h}(\bar{t})=I_{l}$ in (8) at $\bar{t}=\bar{t}_{k, h}$ and, $\bar{t}=\bar{t}_{k, l}$ respectively. Then, the inner solutions are responsible for the process of fast switching between low and high concentration states. They have the form:

$\int_{n h, l}^{n} \frac{n}{a(n) I_{k-1, h, l}-n}= \pm \frac{\bar{t}-\bar{t}_{k, h, l}}{\beta}$,

where $n_{h}=n_{H}\left(I_{h}\right), n_{l}=n_{L}\left(I_{l}\right)$. The sign «+» corresponds to the inner solution determining switching from the low concentration to the high concentration branches at $I_{k-1} \approx I_{h}$, while the sign «-» is for the inner solution joining high concentration to low concentration branches at $I_{k-1} \approx I_{l}$.

Now we obtain the evolution of the photo-excited plasma in the $k$ th QW as follows. If the intensity of the light reaching this well, $I_{k-1}$, increases from zero, the well is in the low absorption state with plasma concentration $n=n_{L}\left[I_{k-1}(\bar{t})\right]$ until $\bar{t}=\bar{t}_{k, h}$. In a vicinity of $\bar{t}_{k, h}$ a fast switch-on process to the high absorption state with $n=n_{H}\left[I_{k-1}(\bar{t})\right]$ occurs according to (11). A further increase in the intensity leads to an increase in the plasma 


\section{A. Kochelap et al.: Switching-on and -off dynamics of MQW structures ...}

concentration and the absorption in accordance with the high absorption branch. If the intensity reaches a maximum and then decreases, the $k$ th QW remains in this state up to time $\bar{t} \approx \bar{t}_{k, l}$, when a fast switch-off process (described by (11)) to the low absorption state occurs. As a result we have dynamic hysteresis for the $k^{\text {th }} \mathrm{QW}$. To be switched-on, the $k^{\text {th }} \mathrm{QW}$ should accumulate plasma concentration up to $n_{h}$. The necessary time is estimated as $\Delta \bar{t}_{\text {on }} \approx \beta\left(n_{h}-n_{l}\right) / n_{h}$. The switch-off process occurs in a time $\Delta \bar{t}_{\text {off }} \approx \beta$. Important characteristics are the changes in the intensity $I_{k-1}$ during these fast stages. These changes are of the order of

$$
\begin{aligned}
& \Delta I_{k-1}^{o n} \approx \Delta \bar{t}_{o n}\left(\frac{d I}{d \bar{t}}\right)_{t_{k, h}} \approx \beta \frac{n_{h}-n_{l}}{n_{l}} I_{h}, \\
& \Delta I_{k-1}^{o f f} \approx \Delta \bar{t}_{\text {off }}\left(\frac{d I}{d t}\right)_{t_{k, l}} \approx \beta I_{l} .
\end{aligned}
$$

Let us suppose that $\Delta I_{k-1}^{o n}$ and $\Delta I_{k-1}^{o f f}$ are small in comparison with the steady state attenuation in a single QW, $a\left(n_{k}\right) I_{l, h}$. These conditions can be rewritten as

$\beta \frac{n_{h}-n_{l}}{n_{h}} \ll<, \quad \beta<a$.

If inequalities (12) are fulfilled, the times at which different QWs switch are well separated. Indeed, according to the above analysis, at the time $\bar{t}_{1, h}$ when the very first QW switches to the HA state, the adjacent QWs follow adiabatically the intensity $I_{1}$. At $\bar{t}_{2}$, the second QW switches-on, but the following QWs with $k>2$ still follow adiabatically the intensity $I_{2}$, and so on. Thus we find that the MQW structure switches on sequentially as the intensity of light increases.

The reverse process - switch-off of the structure occurs similarly. It starts when the intensity of light at the last QW in the HA state reaches the value $I_{r}$. Then a step-like «switch-off» wave propagates towards the first QW.

The step-like propagation of a «switch-on» wave through the structure was also found by direct numerical simulations. The results were obtained for a MQW structure with $N_{\omega}=24$ illuminated by an incident light of intensity

$$
I_{0}(t)=I_{s}(1-\exp (-\beta t)) .
$$

In fig. 4 we present numerical results for $I_{s}=1.25 \times I_{h}$ and $\beta=0.1$. The intensities $I_{k-1}$ are shown as functions of $t$ for $k=1 \ldots 8$. Nonmonotonic and sawtooth-like $I_{k-1}(t)(k=2 \ldots 8)$ are due to the step-like switch-on of the QWs. In fig. 4(b), the concentrations $n_{k}(t)$ of the first nine QWs are shown. We clearly see that seven QWs switch in succesion to the HA state at different times, while the other QWs remain in the LA state. As $t \rightarrow \infty$ the input intensity

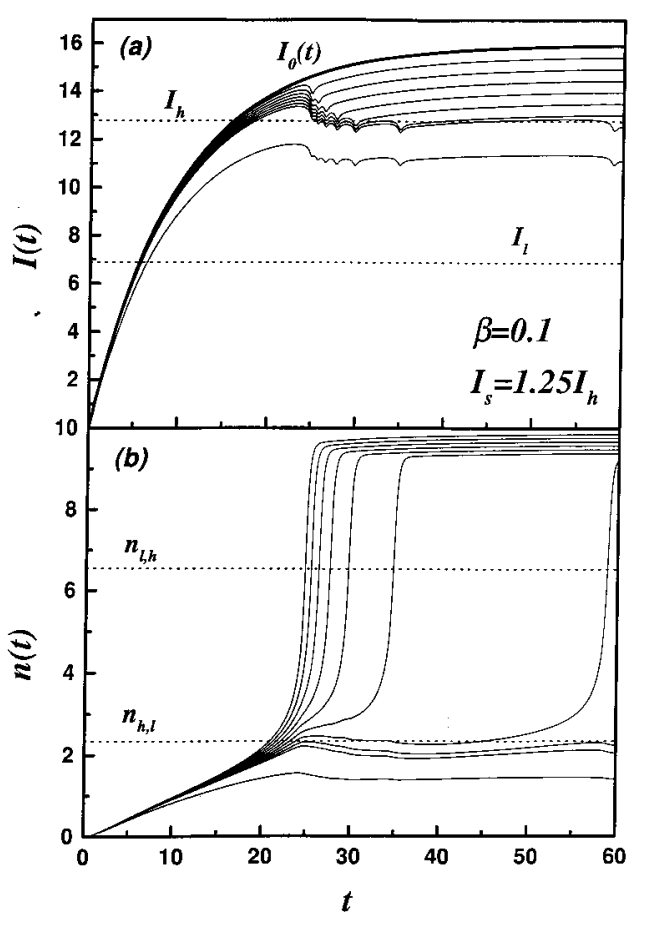

Fig. 4. Dynamics of the MQW structure illuminated by the light intensity given by (12) 1 at $I_{s}=1.25 \times I_{h}$ and $\beta=0.1$. The critical intensity values $I_{l}$ and $I_{h}$ are shown. (a) shows the incident intensity, $I_{0}(\mathrm{t})$, and the intensities illuminating the first eight QWs and the transmitted intensity (the lower curve). (b) shows the plasma concentration for the same wells. The first seven QWs are switched to the HA state.

saturates and the structure evolves to the steady state distribution presented in case (i) of fig. 3(a).

The step-like character of the switching process obtained above in the limiting case (7) occurs also when $\beta \sim 1$. This is illustrated by the numerical simulations presented in fig. 5 for a $I_{0}(t)$ given by (12) with $I_{s}=1.25 \times I_{h}$ and $\beta=1$. As $t \rightarrow \infty$ illumination with such an intensity results in a steady state distribution with eight QWs in the HA state (see case (ii) of fig. 3(a)).

Increasing the light intensity leads to a larger number of QWs switching to the HA state. In fig. 6 we present simulation results for an incident intensity given by (13) with $I_{s}=2 \times I_{h}$ and $\beta=0.1$. From this figure we can see that the step-like switching process now involves all the QWs. For this intensity the final distribution corresponds to the steady state presented in fig. 3(b). It is interesting to note that the output intensity is close to the value $I_{h}$.

For a pulse illumination with a maximal intensity above $I_{h}$ both processes - switch-on and switch-off - take place. Using a gaussian pulse:

$I_{0}(t)=I_{\max } e^{-(\beta t)^{2}}$

we found step-like switch-on and switch-off waves. In fig. 7 we plot calculations of the transmitted intensity $I_{\text {out }}$, as a function of the incident light intensity $I_{0}$. The results were obtained for $I_{\max }=1.25 \times I_{h}$ and $\beta=0.1$. Instead of a smooth 


\section{A. Kochelap et al.: Switching-on and -off dynamics of MQW structures ...}

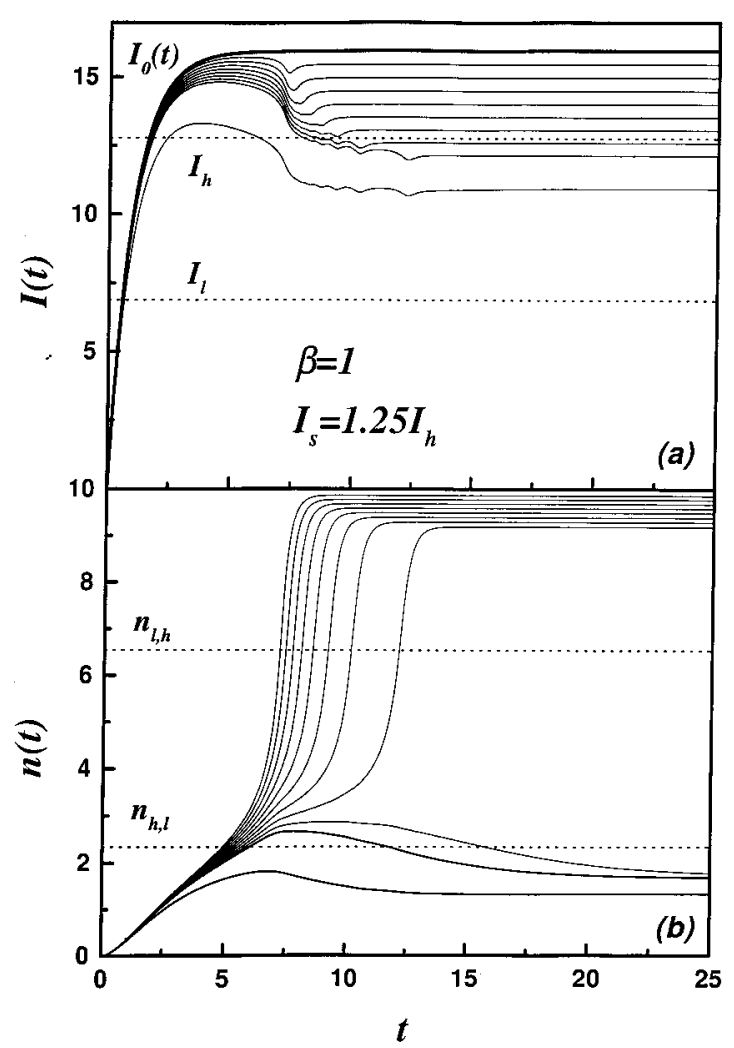

Fig. 5. Same situation as of fig. 4 with $\beta=1$. Eight $\mathrm{QWs}$ are switched to the HA state at $\tau \rightarrow \infty$.

hysteresis loop, we find one with a fine structure corresponding to the switch-on and switch-off processes.

It is interesting to recall that optical bistability with increasing absorption occurs also in bulk-like semiconductors. Different mechanisms underlying this type of bistability and different spatio-temporal patterns have been studied in [13$16]$. For such systems, it has been found $[17,18]$ that if the diffusion of the photogenerated carriers is suppressed, switch-on and -off processes occur in a step-like propagating wave, which is quite similar to the above discussed results.

In conclusion, we have studied the dynamics of MQW structures under bistable electro-optical absorption. We have formulated a model self-consistently describing the electron and hole wave functions and energies, and the processes of absorption, generation of the plasma and intra-well relaxation. The inter-well transfer has been supposed to be negligible. Under these conditions, steady state distributions of the intensity and plasma concentration are multistable. For time-dependent incident intensities we have found that switching processes between possible states of the structure occur as a result of sequential step-like switching of individual QWs. This leads to a characteristic behavior of the transmitted intensity and input-output dependencies. It is worth to notice that due to

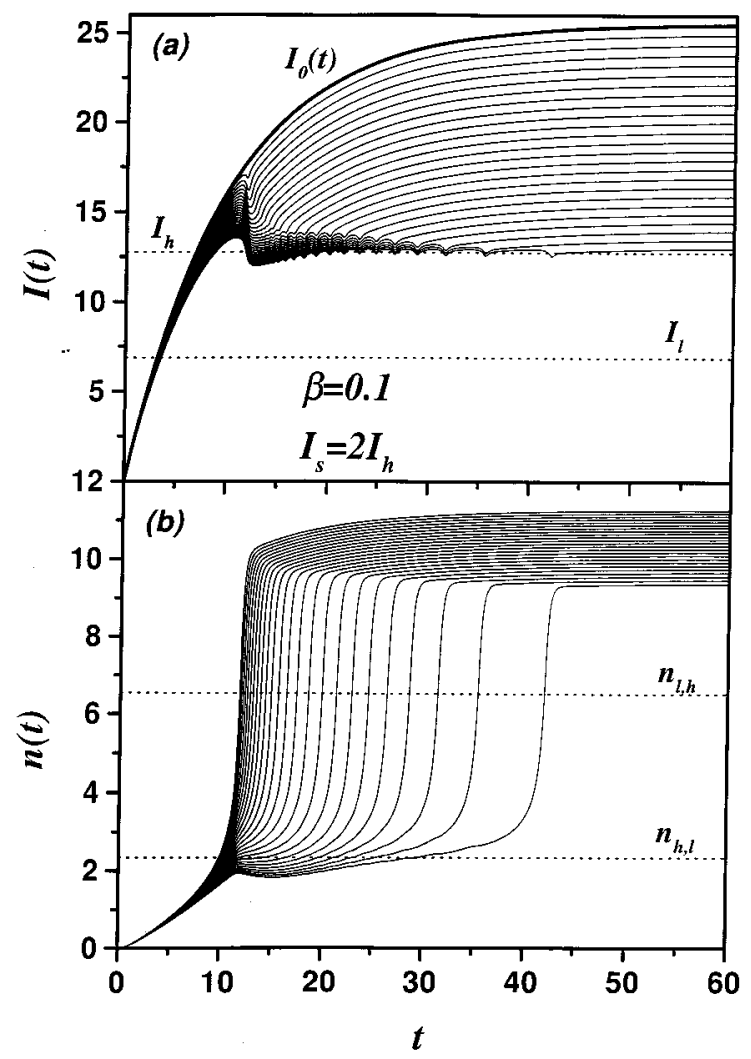

Fig. 6. Same situation as of figures 4 and 5 with $I_{s}=2 \times I_{h}$ and $\beta$ $=0.1$. All the wells are switched to the HA state. Only wells with an odd index are shown.

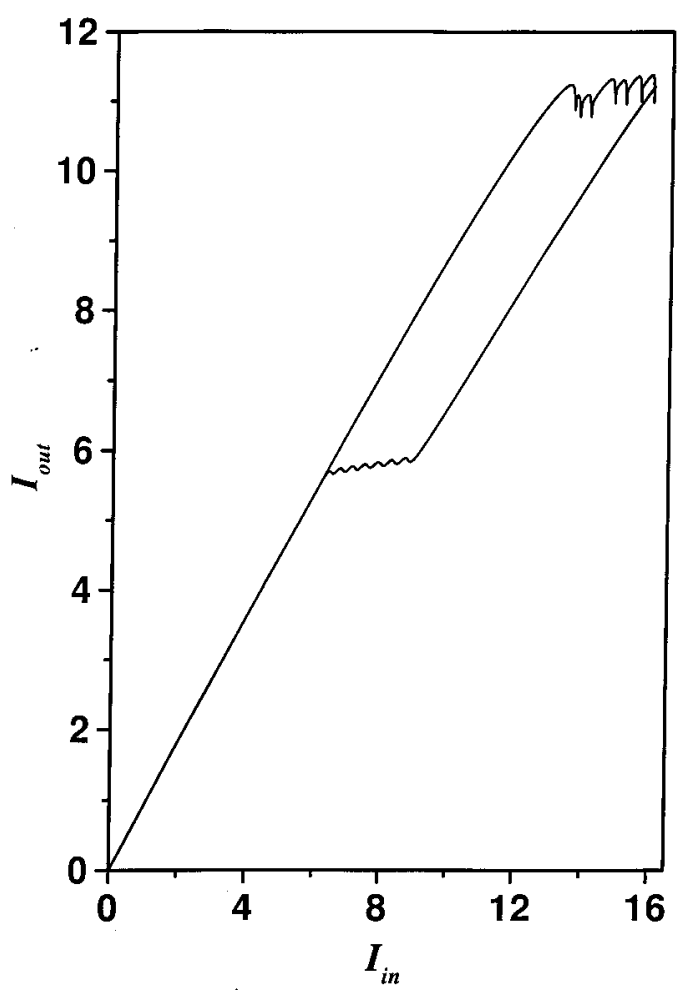

Fig. 7. Output versus input intensity for the pulse given by (14). 


\title{
V. A. Kochelap et al.: Switching-on and -off dynamics of MQW structures ...
}

step-like switching we can use these MQW structures for the conversion of an analog optical signal to digital (optical and electrical) signal(s).

\section{Acknowledgements}

We are indebted to the Direccin General de Enseanza Superior (Spanish Ministry of Education) for sabbatical support (VAK) and for financial support through grant PB94-0375. One of us (CAV) acknowledges the support of the Fundacin General de la Universidad Carlos III de Madrid.

\section{References}

1. D. A. B. Miller, D. S. Chemla, T. C. Damen, T. H. Wood, C. A. Burrus, A. C. Gossard, and W. Wiegmann, IEEE J. of Quantum Electronics, 21(9), p. 1462 (1985).

2. S. Schmitt-Rink, D. S. Chemla, and D. A. B. Miller, Adv. Physics 38(2), pp. 89-188 (1989).

3. A. L. Lentine, H. S. Hinton, D. A. B. Miller, J. E. Henry, J. E. Cunnningham, and L. M. F. Chirovski, IEEE J. of Quantum Electronics, 25(8), p. 1928 (1989).

4. D. A. B. Miller, Opt. Quantum Electron., 22, S-61 (1990).
5. Y. Abe and Y. Tokuda, Appl. Phys. Lett. 63(24), p. 3259 (1993).

6. J. Couturier, P. Voisin, and J. C. Harmand, Appl. Phys.Lett. 64(6), p. 742 (1994); Journal de Physique, Vol. 3-C5, p. 253 (1993); Semicond. Sci. and Technol., 10, p. 881 (1995).

7. R. Merlin, in Spectroscopy of Semiconductor Microstructures, NATO - Series B, Physics, 206, p. 347 (1989).

8. R. Merlin, N. Mestres, A. McKiernan, J. Oh, and P. K. Bhattacharya, Surface Science 228, pp. 88-91 (1990).

9. R. Merlin and D. A. Kessler, B 41(14), pp. 9953 (1990).

10. J. A. Trezza, M. C. Larson, S. M. Lord, and Jr. S. J. Harris, J. Appl. Phys. 74, p. 1972 (1993); S. Scandalo and F. Tassone, (b) 173, p. 453 (1992).

11. L. L. Bonilla, V. A. Kochelap, V. N. Sokolov, and C. A. Velasco, (b) 204, pp. 559-562 (1997).

12. J. Kevorkian and J. D. Cole, Perturbation Methods in Applied Mathematics, Springer, New York, (1981).

13. V. A. Kochelap and V. N. Sokolov, (b) 146, p. 311 (1988); V. A. Kochelap, Yu. L. Mel'nikov and V. N. Sokolov, Sov. Phys. Semicond. v.16, p. 746 (1982).

14. K. Bohnert, H. Kalt and C. Klingshirn, Appl. Phys. Lett. 43, p. 1088 (1983).

15. H. Rossmann, F. Henneberger and J. Voist, (b) 115, p. 1063 (1983). F. Henneberger and H. Rossman, (b) 121, pp. 685 (1984).

16. H. E. Schmidt, H. Haug and S. W. Koch, Appl. Phys. Lett. 44, p. 787 (1984)

17. S. W. Koch, H. E. Schmidt and H. Haug, Appl. Phys. Lett. 45, p. 932 (1984); M. Lindberg, S. W. Koch and H. Haug, A 33, p. 407 (1986).

18. H. M. Gibbs, G. R. Olbright, N. Peyghambarian, A 33, p. 692 (1985).

\section{ДИНАМІКА ВКЛЮЧЕННЯ ТА ВИКЛЮЧЕННЯ БКЯ СТРУКТУР 3 БІСТАБІЛЬНИМ ЕЛЕКТРООПТИЧНИМ ПОГЛИНАННЯМ.}

\author{
B. О. Кочелап \\ Інститут фізики напівпровідників НАН Украйни
}

Л. Л. Бонілла, К. А. Веласко

Мадридський університет ім. Карлоса ІІІ, Іспанія

Резюме. В роботі досліджена динаміка включення-виключення у несиметричних (зміщенних) БКЯ структурах, де кожна яма показує оптичну бістабільність у області інтенсивностей світла $\left(I_{l}, I_{h}\right)$. Детально проаналізовано БКЯ структури 3 незначним переносом між ямами. Знайдено, що механізм включення складається 3 часової послідовності , коли кожна квантова яма (КЯ) переходить в сильнопоглинаючий стан. Таким чином, в структурі розповсюджується ступінчата хвиля. Процесс виключення нагадує зворотню хвилю, яка розповсюджується в протилежному напрямку и ступінчати процеси у спаді концентрації плазми. Ці ефекти можуть бути використані для перетворення аналового оптичного сигналу у цифровий (оптичний та електричний).

\section{ДИНАМИКА ВКЛЮЧЕНИЯ И ВЫКЛЮЧЕНИЯ МКЯ СТРУКТУР С БИСТАБИЛЬНЫМ ЭЛЕКТРООПТИЧЕСКИМ ПОГЛОЩЕНИЕМ.}

\section{В. А. Кочелап}

Институт физики полупроводников НАН Украины

\section{Л. Л. Бонилла, К. А. Веласко \\ Мадридский университет им. Карлоса ІІІ, Испания}

Резюме. В настоящей работе исследована динамика включения-выключения в несимметричных (смещенных) МКЯ структурах где каждая яма обнаруживает оптическую бистабильность в области интенсивностей света ( $\left.I_{l}, I_{h}\right)$. Детально проанализированы МКЯ структуры с незначительным переносом между ямами. Обнаружено, что механизм включения состоит из временной последовательности, когда каждая квантовая яма (КЯ) переходит в сильнопоглощающее состояние. Таким образом, в структуре распространяется ступенчатая волна. Процесс выключения напоминает обратную волну, распространяющуюся в противоположном направлении и ступенчатые процессы в спаде концентрации плазмы. Эти эффекты могут быть использованы для преобразования аналогового оптического сигнала в цифровой (оптический и электрический). 